found the canals full of a thick bile, and in one case implicating the supra-hepatic veins; this fluid was albuminous, the liver being fatty. In chlorosis, the bile is more tasteless and pale; while in scorbutus, the gallbladder is distended with a livid green fluid. The exaggerated action of the liver after the bites of poisonous animals, producing a polycholia, may be regarded as an eliminatory effort of nature. In various pernicious fevers, and in the plague, the properties of the fluid have been observed altered, and in Asiatic cholera the bile undergoes inspissation.

2. Influence of the bile on different diseases. A mere excess of bile may impress on diseases a character foreign to their nature, and may furnish special indications. From this source a form of pneumonia, of which Stoll has furnished the completest description, seems to derive its peculiarities. At one period it would seem to have prevailed with great intensity over a large portion of Europe, if we are to judge from the 80 memoirs referring to it, reviewed by the Soc. Roy. de Méd. during 1782-4. Doubtless much exaggeration prevailed; and so seldom is bilious pneumonia now seen in Paris, that M. Grisolle has only met with ten cases in six years. Still its occasional well-marked existence cannot be doubted. Certain febrile affections, too, may acquire a bilious character, not as a mere consequence or complication, but as an essential feature; and M. Delaroque even attributes the production of typhoid fever to the action of degenerated bile upon the intestinal canal. It is, however, especially in the bilious remittents of hot climates, that we observe the action of this fluid.

\title{
Art. VIII.
}

1. Females and their Diseases; a Series of Letters to his Class. By Charles D. Meigs, M.D., Professor of Midwifery and the Diseases of Women and Children in the Jefferson Medical College at Philadelphia, \&c. \&c.-Philadelphia, 1848. 8vo, pp. 670.

2. A Practical Treatise on the Diseases peculiar to Women. Illustrated by Cases derived from Hospital and Private Practice. By SAMued Ashwell, M.D. Third Edition.-London, 1848. 8vo, pp. 772.

THE works which we have to review emanate from two physicians, each of whom has filled the obstetric chair in a school of medicine, - the one at Guy's Hospital, and the other at the Jefferson Medical College, Philadelphia ; and from whose position and opportunities we have a claim to anticipate a full exposition of what is known upon the subjects they write upon, with such a masterly and mature judgment upon the diseases they describe, as will strengthen and confirm the views of the practitioner, and guide and counsel the inexperienced student. In saying this, we are not placing the standard for our criticism higher than the authors have themselves raised it; nor have we extended the scope of their undertakings. They have both arrived at that professional age, when visionary or immature views are generally forced to yield before the exacting influence of a large responsible daily practice; and it is this conviction which confessedly has been, with both of them, a leading-and, we would add, a praiseworthy-motive for their publishing. But although Dr. 
Meigs and Dr. Ashwell may be alike in these particulars, their respective works have marked individual peculiarities. Dr. Ashwell's treatise has been before the public for the last four years, and has reached the third edition; although it ought to be noticed, that the second edition was very limited in number, and without emendation or correction by the author worthy of notice. The present edition, however, has some important additions, which render the work more complete. The treatise is divided into two main sections; the first comprising the functional, and the second the organic, diseases of the Uterus. In the former, besides the ordinary functional disorders, are included the subjects of Chlorosis, Leucorrhœa, Hysteria, and Irritable Uterus; and in the latter, in addition to the recognised organic diseases of the uterus and ovaries, there are the Displacements of the Womb, the Diseases of the External Organs, and two Essays, - one on "Premature Labour in Pregnancy complicated with organic disease," and the other "On the Morbid Consequences of Undue Lactation."

We need here only observe, that, for extent of subject-matter, for copiousness and variety of illustration, for authentic practical knowledge, and for vigorous style of writing, the book is not excelled by any on the same subject; and this we say, without prejudice to our free and unbiassed commentary on some of the views and opinions which it contains.

We must not, however, dismiss our general review of Dr. Meigs's work with so short a notice. We may say, however, that he has written on most of the important diseases of the uterus, both functional and organic; and that several letters are devoted to subjects which are generally included in purely obstetric treatises, such as the Disorders of Pregnancy, Abortion, Flooding after Delivery, Puerperal Fever, \&c. In this respect he appears to have followed M. Colombat, whose prolix and cumbrous work he translated in 1845 ; and as the annotations he then contributed to it formed, in our mind, the best part of the work, we were induced to urge on him to publish an original treatise on the same subjects. It required but very little knowledge of Dr. Meigs, to perceive that, if he did write at all, he would indulge in some peculiarities, if not of opinion, at least of diction; but we were hardly prepared for such a strange infringement upon the established rules of lexicography and good taste, as we have here before us. If, as a French author says, the style is the man, Dr. Meigs ought to have a large share of what is flimsy and fanciful, combined with the more substantial qualities which have made him a sound and successful obstetric physician. The book is over-garnished, and is filled with mannerisms, not unmixed with extravagance.

Of this Dr. Meigs is evidently conscious ; for in his Introductory Letter to a Friend, he requests him to say whether he has "committed an unpardonable breach of the forms of our science by writing with such a freedom and abandon." For our own part, we are quite willing to sympathise with even a medical author, who endeavours to lighten the labour of his readers by blending a little literary gaiety and humour with the bald facts of which his art is constructed. But while we welcome this ingredient in a scientific work, if nicely subdued, it becomes offensive and reprehensible when too conspicuous, or if it be paraded-as is too often the case with Dr. Meigs - with a garish ostentation. It is only necessary to read the first half-dozen letters, to be convinced that Dr. Meigs has 
some very peculiar methods of engaging the attention of the young gentlemen to whom he addresses them. At one time he startles them with a proposition, such as the following - "stroma is sex ;" at another he is quite overwhelming with a torrent of fine writing-here coining a word, and there barbarizing another; and then he launches into an imaginary conversation with a patient-with some hypothetical "Miss Mary," or " my darling Helen," - as his way of teaching the diagnosis and management of disease. Now, in all this there is a little personal portraiture; and we soon learn to recognise that Dr. Meigs is a gentleman of versatile talents and varied knowledge, of the most enchanting manners, well dressed, of great conversational powers, fluent, quite at his ease, and very striking; in short, a most agreeable, well-educated, good-humoured man - the very beauideal of an obstetric dandy. But, while noticing thus prominently these failings of Dr. Meigs, we are anxious to assure our readers that they are only on the surface; and that the body of the book is worthy of attentive consideration, and is evidently the production of a clever, thoughtful, and sagacious physician. We should, indeed, have willingly passed by these whimsical conceits of our author, in consideration of the high merit which really belongs to him; were it not that, as displayed by a teacher, in which capacity he writes the letters, they have a particular significance. It requires but little acquaintance with that miscellaneous race of young men, the students of a medical college, to know that many are more allured by the gaudy colouring of a turgid style of composition, than by the hard thought and patient observation which may be concealed beneath or obscured by it; and that many a man may live under the delusion that, in emulating the former, he has acquired the latter. But, independently of this, we should unaffectedly regret if Dr. Meigs's formula for catechising patients should be too implicitly followed by his pupils; for we think it more than an imaginary danger that such endearing titles as " my dear child," " my darling miss," \&c., should be imitatively lisped, or, it may be, improved upon, by the rising race of Philadelphian practitioners, to the scandal of obstetric practice, and the offence of well-bred female modesty. Perhaps in thus alluding to this subject, we may be thought to be prudishly fastidious; but we more than suspect that the same kind of address is prevalent enough on this side the Atlantic; and we are quite sure that it ought to be discountenanced and rejected, and settled as a part of the peculiar vocabulary of that venerable body of matrons, the monthly nurses.

As a preliminary inquiry into the functional disorders of the uterus, the authors consider the subject of Menstruation. The recent physiological researches which have connected the menstrual periods with the spontaneous discharge of ova and the act of impregnation, are alluded to by Dr. Ashwell, and taken up with some enthusiasm by Dr. Meigs. It is to be regretted that Dr. Ashwell has not entered more fully into the subject; especially as we gather, from what little he does say, that he is at variance with the now prevalent opinions of Bischoff, Lee, and others. He relates three cases, in which he examined the ovaria of women who had died during the menstrual flow, in neither of whom was there the physical evidence of a maturated or rent Graafian vesicle; and he concludes generally, that it is by observations on the human female, and not on animals during the period of heat, that this theory ought to be esta- 
blished. Dr. Meigs, on the contrary, in a well-written chapter on the menstrua, thus summarily expresses his opinion :

"A healthy woman matures, and deposits an ovum every twenty-eighth day, from the age of 15 to that of 45 years, failing only in case of pregnancy and lactation, and sometimes not even then. She sometimes suffers an arrest of the force during lactation, yet in the majority even that arrest is but of short duration, and in many it does not take place at all. The closing stage of the process of maturing and depositing, or discharging the ovum, is attended with a discharge of bloody fluid from the genitalia, which is called menstruation, because it takes place once a month." (p. 385.)

For our own part, we are quite disposed to believe that the establishment of puberty, and the flux of blood from the womb once a month, depend on the organic perfection and functional activity of the ovaries; but we have the same hesitation as Dr. Ashwell in believing that precise dogma, which affirms that an ovum is periodically cast off at each menstrual period, and that observations, which have been made on the lower animals during their œstrum, are to be regarded as strictly applicable to the catamenial periods of women. Passing by several minor considerations, there is this great practical anatomical difficulty to be yet overcome, which hitherto, we feel confident, has not been satisfactorily accomplished ; viz. the presence of a true corpus luteum when we know, from the fact of impregnation, that an ovum has been cast off; and the absence of any such body after the ordinary menstrual periods. We do not deny the spontaneous discharge of ova in the rabbit, sow, \&c. \&c., at the time of heat; or that this periodical oviposition is followed by luteal bodies in their ovaries; but we deny that any such bodies are to be seen in the ovaria of the human female as the simple result of menstruation. We have had ample opportunities of comparing the true corpora lutea, which have followed on conception in the female, with the vacated follicles or the extravasated blood - which may accompany the ovarian congestion of menstruation, and form false corpora lutea. But the two are in no notable particular alike; indeed, they are perfectly dissimilar; and, in our mind, it has yet to be shown that the human female is the subject of a periodical œstrum once a month, and that at this time vesicles are maturated and an ovum discharged, which may be impregnated during its slow progress through the sexual organs, whenever it may come in contact with the semen. We believe that about eight days is given for this transit of the ovum, which, in fact, limits the period of conception to this time after the menstrual period. And yet the Jewish women, who are bound to observe continence from sexual intercourse for just this time after menstruation, are notoriously prolific as a race! We are quite disposed to concur with Dr. Ashwell, that it is an arbitrary assumption to make experiments on the lower animals apply directly to the human female; and that, in drawing inferences respecting a peculiar function of the latter, the only observations which are conclusive, are those derived from the appearances of the ovaria and sexual organs when performing this function.

We may incidentally notice an opinion of Dr. Meigs, that the corpus luteum is a true vitellary body,-a discovery which he thus announces: "These researches," says he "suffice to convince me that the yelk of eggs and the yellow matter from a corpus luteum are of the same apparent constitution, form, colour, odour, coagulability, refractive power, and 
microscopic appearance." It is not our purpose to enter minutely into the observations of other physiologists on the structure of the corpus luteum, or to attempt the full refutation of this notion of Dr. Meigs; but shall content ourselves with remarking that, whatever may be the similarity in elementary composition-whether chemical or organic--between the vitellus and the corpus luteum, there can be no real analogy, either structural or functional, between them; seeing that the one is within, and the other without, the ovum; and that the ovum of the mammalia has as true a vitellus as that of the oviparous animal, the embryo being nourished by it alone during the earlier stages of its development, to which operation it is evident that the corpus luteum can contribute nothing whatever.

Dr. Ashwell's chapter on Chlorosis, with its complications, is very full and practical, and will amply repay a careful perusal; while Dr. Meigs's notice of it is included in a lengthy supposed conversation with a mother, about her daughter Mary, in which the Doctor allows his imagination to have full scope, but still without failing to put forth the prominent features of the complaint, with its pathology and rational therapeutics. We need scarcely observe, that this blood-disease is, by both, treated on those general hygienic principles which are now so well understood; the effect of which is, to improve nutrition, enrich the blood, and impart tone and vigour to the enfeebled constitutional powers. Among special remedies, the iodide of iron is spoken of as most valuable.

Several very interesting cases of organic amenorrhœa resulting from the absence of the uterus, and of retention of the menses from congenital and artificial closure of the vagina, are related by both our authors. The occasional success of surgical operations in opening a passage through the closed vagina, and in giving vent to the collected menstrual fluid, is well known, and is illustrated in these works; but it must be admitted that attempts of this kind not unfrequently fail in their object, and expose the patients to the great additional risk of a fatal peritonitis. Case, No. 21 (page 88), in Dr. Ashwell's book, is very instructive on this point.

The two functional disorders which we shall have to consider are, dysmenorrhœa and menorrhagia.

Dysmenorrhoea. Severe pain during the whole or part of the menstrual times is the symptom which has originated this designation dysmenorrhœa; but, as pain is the result of different, and sometimes even opposite, pathological states, the term is liable to confuse disorders; and we find this the case, to a certain extent, in the chapters of both authors. Dr. Ashwell's divisional arrangement of the subject is into neuralgic dysmenorrhœa, plethoric dysmenorrhœa, congestive dysmenorrhœa, and lastly he notices Dr. Mackintosh's cases of dysmenorrhœa dependent on mechanical obstruction. Dr. Meigs makes no division at all; but, in his own discursive mode, brings in something of them all, saying some very good things of each, and in a very descriptive way.

"The pain of dysmenorrhœa is a pain felt in the hypogastric region, in either or both of the iliac regions, in the tractus of the ligamenta rotunda, in the sacral region, in the thighs, and very frequently in the course of the distribution of the obturator nerves. This pain, not unfrequently, extends to the whole belly, and is at times insupportable, from its violence; compelling the patient to lie down in bed, or on the couch; forcing from her both tears and groans, and producing so 
great a degree of restlessness, that she seems to writhe like a crushed worm." (p. 435.)

In this picture perhaps the only omission is in not referring much of the suffering to the ovaria themselves; which, we think, are very commonly implicated in this morbid sensibility.

Referring to Dr. Ashwell's division, which is substantially identical with that of other authors, as Dr. Churchill, \&c., we are quite disposed to concur in the appropriateness of the first and last varieties-the neuralgic and the mechanical.

The subjects and temperament of the neuralgic cases, the character of their suffering, and the absence of any active congestion of the uterus, not to mention the direct indication, and the successful result of their treatment during the intermenstrual times, by steel, quinine, and tonics, - sufficiently characterise this division. The cases in which rheumatism has been supposed to have attacked the unimpregnated uterus, which is alluded to as a positive cause of dysmenorrhoea by Dr. Meigs and others before him, may be allied to the hysteralgic cases, and fairly included under this division. "Do you not perceive," says Dr. Meigs, "that a rheumatic womb is likely to be a painful womb during the menstrual act, and that much of the dysmenorrhoea which it will be your destiny to encounter in your future career as practitioners, will be regarded by you as a form of rheumatism?"' For this disorder the ammoniated tincture of guaiacum has been highly praised by most obstetric practitioners, although Dr. Meigs has not found it so useful. He speaks in high terms of "moderate doses of the golden sulphuret of antimony, combined with camphor, and small doses of opium or morphia a few days previous to the attack of dysmenorrhœa."

Mechanical dysmenorrhoea. Without following the order of Dr. Ashwell's paragraphs, we may notice that he speaks in very equivocal terms of narrowing of the os and cervix uteri as a cause of dysmenorrhœa. He appears to us to entertain the faintest possible perception that it may just be the case ; although even its half-admission in the first part of a sentence is all but smothered before he completes it. "There is no doubt," says Dr. Ashwell, " that dysmenorrhœa has occasionally coexisted with such malformation; but it is by no means certain that it owes either its origin or its continuance to such a state of parts as a cause." And although he quotes Dr. Mackintosh's cases, and others by Dr. Churchill, Oldham, \&c., which were treated and cured by overcoming the strictured os, yet he still shows, in a note in the new edition, that he retains his scepticism. Dr. Meigs, on the contrary, speaks of congenital strictures and flexions of the cervix uteri, as mechanical causes of dysmenorrhœa ; and he expresses, in the following terms, his experience of the employment of dilatation to overcome it, and of the relative value of incising and dilating the strictured part for this purpose.

“Dr. Mackintosh's practice has been used everywhere, and with undoubted success in cases proper for it. Some of the brethren have preferred to Mackintosh's method, the use of a concealed bistoury, which being introduced to the upper extremity of the canal, is slightly disclosed by a spring, and then being withdrawn entirely from the os uteri, has divided the lining membrane of the canal, allowing of a complete dilatation of it. When I was in London, in 1845, Dr. Locock showed 
me one of these instruments, with the effects of which he appeared to have been much pleased in his practice.

"If you have read this book, you must have observed in a former letter, that I strenuously opposed the use of all cutting instruments in the procuring of a requisite dilatation, upon the ground that every division must be followed by a cicatrix, which is, essentially, a morbid existence ; whereas all necessary dilatation can, I am convinced, be as readily and perfectly brought about by a distending. pressure, leaving the part, when the part has been thus cured, without a wound, and in a perfectly healthful and normal state. I repeat it, that a cicatrix is not a normal state; it is always insecure, always dangerous. I therefore profess my adhesion to Mackintosh's practice. My own experience in the use of the bougie, has been to a certain extent satisfactory to me; satisfactory to this extent, that it has not only, in some cases, relieved the woman from distress, pain, and other disorders of menstruation, but that it has cured her of a pre-existing sterility. A woman is likely to be sterile when her uterus is unhealthy, and particularly when it is unhealthy from narrowness of its canal." (pp. 438-9.)

So far as our observation goes in the employment of these means as practised in London, we have no hesitation in saying that they are too frequently had recourse to. The cases of stricture requiring dilatation are very rare indeed; and if the operation were less easy and less concealed, we doubt very much whether it would be employed so commonly as we fear it is. A patient of our own, who has been married for some years, but without having been pregnant, consulted a physician, who, on examination, discovered a small os uteri, which he at once proposed to cut open, with the alleged expectation of curing her sterility and dysmenorrhœa together. He quite overlooked, however, a fibrous tumour that projected from the right side of the body of the uterus, which had been the cause of her suffering. The os uteri in this case was normally small, not strictured; and it is highly unphilosophical to fix a certain size as a standard os uteri, and to open it up if it does not quite reach this standard. Dysmenorrhœa, with a scanty flow of the menses, is sometimes associated with an imperfect development of the entire sexual system; the uterus, like other organs, remaining small. Sterility necessarily coexists with this condition; but the indication here is not to open the small outlet of the womb, but so to invigorate the constitutional power as to excite this important system of organs to more complete evolution. We are ourselves disposed to prefer the dilating process to incision with a bistoury, when the case demands this mechanical treatment; and our own plan has been to pass caoutchouc or silver stems, which are perforated so as to receive an ordinary uterine sound through them, and gradually to increase their size. When passed through the os uteri internum into the cavity of the body of the uterus, the sound may be withdrawn, and the stem left for half an hour or more within the canal. By a repetition of this process, a contracted os uteri may be made patent; and both dysmenorrhoea and sterility occasionally cured.

It is in the two intermediate varieties of dysmenorrhœa, that we think Dr. Ashwell has rather confused this subject. Why should plethora or local congestion be made to designate particular forms of dysmenorrhœe? They may exist in amenorrhœa: they may be present in menorrhagia: they may be found without producing dysmenorrhœa. It appears to us that the existence of these conditions is altogether secondary, and sub. 
ordinate to another morbid condition, which can only be rightly interpreted by examining the membrane that is exfoliated in these cases. Both our authors notice this membrane, and the spurious abortions which occur when it incloses a clot of blood. Dr. Ashwell speaks, in the text (p. 109), of a "modified and low form of inflammation, which produces a false membrane assuming, in some instances, the shape of the uterine cavity, and in others being expelled in detached portions." Dr. Meigs, too, calls this membrane "a plastic deposit like the plastic deposit in croup, which proves the existence of an inflammatory state;" so that both our authors consider the membrane as a false inflammatory membrane, just as the Hunters regarded the decidua vera as a lymphy product. But the important and interesting investigations which proved the decidua vera in pregnancy to be nothing more than a growth of the uterine glands, transforming the thin transparent mucous membrane of the uterus into a rich, soft, raised, vascular, cribriform structure, gave a clue to the pathological comprehension of the membrane cast off in dysmenorrhœa. Denman and Dr. Montgomery have very well described the physical character of this membrane; although the differences pointed out by the latter between it and the decidua vera, which are quoted at length by Dr. Ashwell, may, we think, be rather ascribed to different degrees of development, than regarded as disproving their common origin. There cannot, we believe, be a doubt, in accordance with late investigations, but that the decidua vera of pregnancy and the dysmenorrhœal membrane are structurally identical. And if so, we may gather,-1. That, as in pregnancy ovarian congestion is followed as a sympathetic action by engorgement of the uterus, and by the rapid development of the uterine mucous membrane, so in membranous dysmenorrhœa, a primary morbid irritation and congestion of the ovary calls forth a local determination of blood to the womb, congesting it, and occasions a similar enlargement of the uterine follicles:2 . That as the mucous membrane of the uterus, when thus altered by the new glandular growth, never shrinks again, but is cast off as a decidua, leaving the uterus bared of mucous membrane until it is reformed, so in dysmenorrhœa, this change of the membrane is necessarily followed by its exfoliation, whether in shreds, or films, or more perfect moulds; and that this detachment, or as Müller has described it, this moulting, of the mucous membrane recurs again and again at the monthly period. We might easily follow out the analogy between the two, into the kindred sympathetic affections which attend on each, were not our object at present to correct what we believe to be a somewhat singular misconception to the original and identical opinions of Drs. Simpson and Oldham on this subject, which he characterises as new and extraordinary, and he says
that-

"One would require rigid demonstration before admitting as true that every month, for many years in succession, the uterus, even with all its extraordinary
endowments, should possess the astonishing power to cast off and reproduce-in the short space of a fortnight or three weeks-its entire mucous coat. I would ask the author in what way he supposes this almost miraculous process of separating the mucous from the subjacent muscular tissue is accomplished? I have not forgotten that the union of these structures is so firm, that the most dexterous anatomist cannot separate them uninjured, even by the knife. What may we not

5-III. 
anticipate in reference to this wonderful organ, after the new views of menstruation, and these startling opinions?" (p. 110, Note.)

We trust that what we have said above supplies the simple answer to Dr. Ashwell's question, and will relieve him from the suspicion that these opinions of the formation of the dysmenorrhœal membrane rather overtax the womb, or invest it with miraculous and wonderful endowments. Had he only remembered that it is of the mucous membrane in its changed and altered state that it is declared to be separated and cast off, he could not have fallen into such a palpable error as to confound it with its removal by dissection when clean, thin, and closely attached. He might as well affect to ridicule the spontaneous desquamation of the epidermic coats of the fingers and knees occurring after scarlet fever, because in the natural state this process cannot be accomplished by the knife.

Women who are the subjects of membranous dysmenorrhœa, are liable to have the womb permanently bulky, and eventually displaced. If in the early stages a copious menstrual flux relieves the attendant engorgement, this complication is retarded or even prevented; and the patient is in a far better state than when a gradually augmenting solid hypertrophy accumulates in the body and neck of the uterus. "The woman's womb aches," says Dr. Meigs, “ until nature bleeds her." And we may add, that if nature does not bleed enough, local depletion, by leeches or cupping, with hot hip-baths and mild mercurials, are our principal therapeutic means.

Menorrhagia. Dr. Ashwell's chapter on this subject is more comprehensive than the clever and succinct one of Dr. Meigs, and includes two principal forms of the disease, which are again subdivided. The first form is "Profuse menstruation, either as to frequency of return, or the amount of the secretion, or both, without uterine bleeding." The second form is "Profuse menstruation, accompanied by direct loss of blood from the uterine arteries." Without entering fully into the subject, we may notice at the outset a discrepancy in the opinions of the two authors; which consists in Dr. Ashwell regarding the blood which flows and coagulates as it flows, as something superadded, and distinct from the ordinary catamenial fluid; while Dr. Meigs, more correctly we believe, regards the menses as blood altered only by its admixture with vaginal mucus. We cannot help transcribing Dr. Meigs's first paragraph, which is quite ad rem.

"The word menorrhagia means immoderate flow of the menses; which flow consists of a purer blood than that of the regular catamenial evacuation. The blood is purer, because the impetuous haste of the discharge causes it to come off less mixed with cervical and vaginal mucus and epithelium, than in the more moderate or normal mensual hemorrhage; - and this, I believe, is the only difference between them. As the blood in menorrhagia is less mixed and combined with foreign matters, you would naturally expect to find it to coagulate more readily; while this is quite true, it is not the less true that the real normal menstruous excretion does, in many women, coagulate, without any suspicion of menorrhagia ; and it is reasonable $\grave{a}$ priori, to suppose so, since one woman may be seven days getting rid of her six ounces of fluid, whereas another woman will part with ten or twelve ounces, in three or five days. She who discharges the menses pleno rivo, will be sure to find some coagula; she who passes it slowly away, will find it to sink into her napkins without a clot, - for her blood has time to be much combined with mucus and epithelium." (p. 424.) 
The pathology and treatment of menorrhagia are well discussed by both the authors; although we must own our surprise at Dr. Meigs's treatment of a young lady, æet. 18, whom he allowed to flood on during the whole night, "suffering her to faint again and again," before he would use the tampon. His commentary on the case is "I do not regret that I allowed her to bleed so much and so long. I would permit another young lady to do the same thing; because, having confidence in the power of the tampon to suppress such a flooding, I would let her go very far towards a dangerous state, rather than subject her to the mortification of the surgical intervention." We must say that we have rarely met with such a cool determination. Surely Dr. Meigs grievously underrates the serious results of such a loss of blood in so young a subject,--the chances of subsequent feeble life and health, with the danger of the invasion of organic disease : to risk it all for a mere feeling, which any obstetric physician ought to know how to mitigate or overcome,-when he confessedly has the means in his power to arrest it. And this is what Dr. Meigs calls being thoughtful and circumspect, and not "snapping at a conclusion like a duck at a June-bug"'!

We pass by the chapters on Leucorrhoa and Hysteria in Dr. Ashwell's work, with simply the remark that, as our more precise knowledge of disease has shown leucorrhœa, in the varieties of discharge mentioned by Dr. Ashwell, to be mainly symptomatic; so it would almost have been better had the author in this new edition either greatly compressed it, or sunk it in the description of the structural disorders which it accompanies. Dr. Meigs does not consider leucorrhœa separately.

On the organic diseases of the womb. As an important practical preface to the consideration of the special organic diseases of the womb, Dr. Ashwell gives a chapter with the very comprehensive title of "General remarks on the history, symptoms, diagnosis, pathology, and prognosis of the organic diseases of the uterus." It appears to us that this chapter, for the student at least, wants both condensation and precision. It is discursive, running over, in an allusive manner, various points of pathology, \&c., and omitting some of the most important elementary principles of diagnosis.

The second section is on the "Examination by touch;" and this, which is a principal source of diagnosis, is defective in that form of precept which is most wanted as a clinical guide to the student or practitioner. Dr. Ashwell details only one mode of examination, so far as the position of the patient is concerned, viz. when in the "recumbent posture, on her back, and without stays, the shoulders elevated, and the lower extremities flexed upon the trunk;" and this mode of diagnosis is applied only to one class of cases connected with the body of the womb, viz. those in which there is increased bulk; and these are illustratively supposed to be confounded with pregnancy. Even taking this example of bulk, no one knows experimentally better than the author, that the physical diagnosis between tumours of or in the womb and pregnancy, and between one tumour and another, is greatly facilitated by the patient being placed in different positions, according to the circumstances of the case ; that what may be ascertained in one position with one finger, may be overlooked in another position with another finger, and so on. It is true, that all which is to be learned on this subject is, in one way or 
another, expressed or implied in detached observations throughout the work; but our objection is, that they are not to be found in a section devoted expressly to the subject, and that this section is proportionately obscure. If we might venture to make a suggestion to Dr. Ashwell, it would be, in any future edition, to put down in so many paragraphs the mode of examining patients, - in the recumbent posture on the back, - on one or other side, - when the pelvis is inverted, - and in the standing posture, with the advantages, whether direct or incidental, which are to be gained by each; and in doing so, not to be afraid to express plain rudimentary facts. We think, too, that the kind of running commentary which he has made on the diagnosis of pregnancy, ought-at least for the purposes of instruction - to be so rearranged, as to present the marks of diagnosis in a more lucid and appreciable shape. The subject itself is almost too extensive to be discussed in this way; and the details of diagnosis, for practical purposes, ought to be fully described, or so grouped together as to present the points of contact in as defined a way as the varieties of tumour or solid enlargement will allow. The evil is, that if the description stops short of these details, or includes them only in part, it is, so far as its instructive adaptation for clinical purposes is concerned, rather embarrassing than otherwise; and we must confess, without criticising this section of the chapter more minutely, that such is the impression which its perusal has left on our mind. The remarks on the diagnosis of diseases of the os and cervix uteri, especially the paragraph on the congenital differences in these structures, are interesting. The author remarks, in reference to vaginal investigations, that " prolonged examination is generally unnecessary; and without arbitrarily limiting the time, such an inquiry ought never, as a general rule, to exceed a few minutes," - an observation, in the truth of which we fully acquiesce.

The use of the speculum and stethoscope, or, in other words, the mediate use of the eye and ear, are discussed as important auxiliaries to the diagnosis of uterine disease. The author does not speak of the best position of the patient for the employment of the speculum; nor does he give any particular preference to one kind of speculum over the other. He thinks, however, that there is no need of division in the cylinder, and that the complicated screw is not required: and he certainly rather bewilders the individual choice of an instrument by saying, that "the best and most easily used speculum is made of tin, pewter, steel, or glass." We might, we think, add yet another, in which a glass tube is covered externally with caoutchouc and a bright surface (Mr. Fergusson's speculum, in short); which, in our experience, exceeds all others in its cleanliness, simplicity and cheapness of construction, and easy application. Its introduction is greatly facilitated by the addition of a smoothly-rounded wooden stem, which, when passed through the cylinder, projects beyond the margin, and prevents the edge either catching or hurting the structures at the vulva. Dr. Ashwell, while fully alive to the great advantages which the speculum offers in the diagnosis of uterine disease, and the precise application of remedies, is yet anxious to damp that enthusiastic use of it which some individuals encourage. He considers that the "hymen in the young, and the shrinking and contraction of the sexual passages in old age, forbid its employment." There are unquestionably some affections of the cervix and os uteri in virgins, attended with a copious leucorrhœal 
discharge, which admit of treatment by the speculum without injuring the hymen, on account of the dilatability which a long-continued leucorrhœa imparts to the sexual passages. But we quite think that any man is guilty of a most reprehensible and indiscreet zeal for the employment of this instrument, who thinks fit to divide the hymen, in order to pass it; and we make this observation designedly, as we see that such a proceeding has recently found an advocate in a contributor to a weekly journal. We have never yet seen a case, which appeared to us to justify in the slightest degree such an operation; and we much doubt whether all the benefit of local remedies might not be obtained by administering them in solution with a syringe, or in small suppositories.

On the employment of the stethoscope as a means of diagnosis, Dr. Ashwell only refers to the similarity of sound produced by the pressure of a fibrous tumour on the iliac arteries, and the placental souffle; and the certain value of the sounds of the fotal heart, as a sign of pregnancy. We might perhaps have anticipated, from a gentleman of Dr. Ashwell's experience, some remarks on the sounds of adhesive bands in the abdomen, and the gurgling noise of the intestines; both of which have practical reference to the diagnosis of ovarian and fibrous tumours.

The uterine sound or bougie is not mentioned in this chapter by Dr. Ashwell, and is altogether omitted by Dr. Meigs; and it is only when speaking of retroflexion of the uterus, that Dr. Ashwell gives his opinion of it. This opinion is very adverse to its general adoption; and the instrument is discarded perhaps too summarily, we might almost say impatiently, by the author. Our own experience of this metallic sound leads us to regard it as one of the most hazardous instruments which has ever been invented; and we think that Dr. Simpson and those who have followed him have something to answer for, in having set forth all that can be said of its real or supposed good, without a corresponding reference to the harm it may accomplish. We have found a very cautious use of it of value in the diagnosis of pelvic tumours; but then it is liable to the chance of puncturing an ovum in the course of these investigations; and we unhesitatingly declare, that to pass it within the uterine cavity, and explore the size, and restore any misdirection of this organ, is very often a painful operation, and is sometimes followed by metritis or pelvic cellular inflammation. The dangers, too, of such an instrument are immeasurably increased by the facility with which it may be passed; and it appears to us to need a far greater habitual caution in its use, than has been thought sufficient by its advocates. It is impossible to be blinded to the fact, that, within the last few years, novel methods of diagnosis and treatment of uterine disease,-almost exclusively of a mechanical kind, have been lavishly and hastily pushed into practice; and although they are very attractive to the inexperienced and enthusiastic, yet, naturally enough, they are looked upon more coldly and cautiously by those, who, like our authors, have learned to weigh the good with the evil which is beside it.

On fibrous tumours of the uterus. Dr. Ashwell distinguishes two varieties of these tumours, classifying them according to their locality : the first class including those which project externally, and carry the peritoneum before them; and the second those which are submucous, and in their growth become invested by the mucous membrane, and deform the 
cavity of the uterus. This distinction is obviously practical; the former being harmful principally by their mechanical interference ; the latter, by the hemorrhage they produce. There are some one or two points connected with these tumours, on which Dr. Ashwell entertains some peculiar opinions. He regards them, for instance, as belonging to "the scirrhous variety of carcinoma," and assigns the following reasons for this view :

"1. They possess the structure of compound adventitious cysts, the basis of this class of heterologue formations. 2. In the colour of the contained mass, and in the arrangement of the membranous septa or bands, the containing tissue, they are identical with scirrhus. 3. In hardness, occasionally justifying the application to them of the term stone cancer, they are not to be distinguished from the varieties of carcinoma already mentioned. 4. They occur very frequently in conjunction with growths of undoubted malignancy in other parts of the uterus. 5. And, lastly, they possess one especial attribute of malignancy, incurability.” (pp. 289-90.)

If, as Bayle declares, the fifth part of women above 35 years of age have fibrous tumours, it certainly assigns, on Dr. Ashwell's supposition, a fearful preponderance of malignant disease to the female over the male sex. We are by no means convinced, however, of their malignant character, by Dr. Ashwell's reasons; and we are more disposed to concur in thinking that " the sum of these reasons does not endow these growths with power to assimilate different tissues to their own substance, nor with the capability to produce that peculiar cachexia by which cancer destroys life."* Perhaps any one who was anxious to establish a general law, that compound adventitious cysts form the basis of cancer-growths, might call the white septa of a fibrous tumour the cyst-walls; although in so doing, we confess that he would have to draw somewhat on the credulity of those who were indifferent to the support of the theory. With our present knowledge, however, of the spread of true cancer by cells, it would have been better to have obtained a microscopic analysis of these tumours; when we think our author would have found them to be composed of a fine unstriped fibre, just like the muscular structure of the uterus itself, without the cells of malignant disease. It appears to us, too, that the fact of their never returning when they have been enucleated, or removed by ligature when polypoidal, or spontaneously detached,-of their enormous volume in some instances, - and the number of them in other specimens,- - besides the compatibility of their existence with good health, - and the freedom of the adjoining structures from cancerous infiltration,-are very decisive proofs of their not coming within the range of malignant diseases.

When speaking of the submucous fibrous tumours, Dr. Ashwell denies the fact that "a genuine hard fibrous tumour ever becomes a pediculated polypus ;" and he recounts several marks of difference between them. "In structure," says Dr. Ashwell, "with some few exceptions, in sensibility, both in the growth and the surrounding parts, and in vascularity, as well as in many other particulars, there is a marked difference between the hard or fibrous tumours of the cavity now described, and polypus." We may mention incidentally, that Dr. Meigs differs from this singular opinion of Dr. Ashwell's, and speaks familiarly of the fibrous polypus. We are not a little surprised to find Dr. Ashwell opposed to what we conceive to be a most familiar morbid demonstration; viz. that some polypi are nothing more than fibrous tumours, which, by the action of the 
uterus have descended lower and lower in the cavity of this organ, dilating the os uteri in their descent, and at length escaping into the vagina or even beyond the vulva. We speak at least from some knowledge of the Museums in London, and with the confidence of personal experience too, when we say that amongst the polypi which have been removed and preserved, such specimens are common; and that their structure is simply the structure of a fibrous tumour and nothing else. Such a tumour has a stalk, more or less thick, of uterine tissue; and an investment either of mucous membrane only, or with a thin shell of the muscular wall of the womb, according to its original position. That such a tumour has structural peculiarities which make it differ from other varieties of polypi, will readily be conceded; and Dr. Ashwell's subsequent observations appear to be based on this admitted fact. "The internal tissue of many polypi," says he, "is spongy and cellular, and copiously permeable by blood; a circumstance scarcely ever appertaining to a hard or fibrous tumour." In this, as in other remarks, our author appears to us to confound the distinctions which mark the varieties of the same disease, with those positive characteristics, either of structure or attendant symptoms, which alone ought to justify their separate classification. A fibrous tumour which has in its descent acquired a stalk, is a bleeding growth, the blood flowing principally from its pedicle : a tumour made up of loose muscular tissue, with large arteries and veins permeating it, and the latter opening on its surface, is another tumour which bleeds like the former one: a third class, originating in the glands of the body or neck of the womb, whether appearing in pendant clusters or with deep channels within them, are bleeding uterine tumours, differing as much in their intimate structure from the second variety, as that does from the fibrous tumour; and yet they all alike are polypi, and admit of cure by removal. Our own experience as to the relative frequency of these varieties, if we except those little nascent polypi from the cervix, is in favour of the fibrous tumour which has been converted by descent into a polypus.

"The want of sensibility," says Dr. Ashwell, "is an almost invariable condition of true polypus; while the hard or fibrous tumour is never entirely bereft of sensation." On this supposed distinction we are bound to say, that if a fibrous polypus is sensitive, which it very rarely is, it is only because it has carried with it in its descent a portion of the uterine structure which still retains its organic sensibility. Generally, however, we are experimentally convinced that no such sensibility is apparent; and that the growth may be cut or pricked with a pin, or noosed by a ligature, without the patient being conscious of pain.

The non-vascular character of fibrous tumours is assigned as a mark of difference between them and polypi. That some polypi are more vascular than others we readily admit; but Dr. Ashwell can hardly have examined the preparations in Guy's Museum attentively, if he denies that fibrous tumours are susceptible of artificial injection. "In the Museum of Guy's Hospital," says Mr. S. Lee, * “there are three preparations illustrating this fact (the vascularity of fibrous tumours); in all these cases the injection has penetrated the morbid mass." The number and size of the blood-vessels, principally arteries, in fibrous tumours, vary extremely in different cases; and are in a great measure proportioned to the con- 
densation of the tissue, and the degree of its infiltration with calcareous matter. We have seen large-sized fibrous tumours very vascular; the trunks of the vessels running in the septa, and dividing freely and minutely in the unstriped fibre of which they are composed; while a belt of largely developed veins surrounds the growth, which rarely can be traced into the tumour itself.

The coexistence of fibrous tumours with pregnancy, which "rarely, if ever," occurs when there is polypus, except where the growth arises from the cervix or os, is cited as a diagnostic difference between the two. On this point we would say, as a general observation, that pregnancy is prevented in both cases; although, if there be no mechanical impediment to the passage of the semen through the tubes, it may by chance occur in either. It appears to us that Dr. Ashwell's assertion of the excessive rarity of polypus in the cavity of the uterus with pregnancy, ought to be modified; for although as a complication of pregnancy it is no doubt unfrequent, yet several cases are on record, which would bear no mean proportion to those related of the same complication from fibrous tumours on the walls of the uterus.

We have thus briefly argued this point with the author, as it is a matter on the right interpretation of which our prognosis in the submucous variety of hard tumours is in a measure founded. In cases of this kind attended with dangerous bleeding, we may at least hold out the expectation that there is yet a chance of cure left in the descent of the tumour, so as to bring it within reach of a ligature. The hemorrhages in this variety of growth are most formidable; and Dr. Ashwell speaks of "palliation in most being all that can be expected." He refers, in the chapter on polypus, to the subject of their enucleation ; but only, however, to deride it ; and on the same point of practice Dr. Meigs speaks of its feasibility, when the growth is in the tissue of the cervix,-where, however, we believe it to be very rarely indeed implanted.

In the treatment of fibrous tumours, the two authors are at variance. Dr. Ashwell speaks in high terms of iodine, which he believes in most cases to be equal to the restraint of the growths; while Dr. Meigs talks of medicine in the following terms.

"People talk of taking medicines for such tumours, and they even take homøeopathic pellets in decillionths of grains! Leaving out of question the unspeakable nonsense of the homœopathic dosings, I see not on what ground they should take even real physic for such complaints, since drugs cannot, and were never designed to heal such tumours and make them return under obedience to the naturaldevelopment laws of the organ, and restore its outline and contexture to a normal form and dimensions." (p. 265.)

We must confess ourselves to have been greatly disappointed in the employment of iodine or the iodide of potassium, which in no instance, out of very many in which we have administered it, has perceptibly diminished or even stayed the progress of the tumours. More good is effected by general means; such as by regulating the diet and exercise, and keeping down by local depletion the tendency to uterine congestion. If there be a medicine which, more than another, will accomplish this diminution or arrest, it is, we confidently believe, the bichloride of mercury, given in small doses, in connexion with tonics, for many months together; but we have never witnessed any such reduction in their size by any plan 
of treatment whatever, as to encourage us to hope that they are capable of removal by any means short of their extirpation. We may incidentally notice an ingenious idea of Dr. Simpson, by which he expected to favour their consolidation, and so stop their increase; by transfixing them with metallic pins, which would keep up an electro-galvanic current through them. Dr. Meigs also talks of tying the uterine arteries, and shaving off the utero-cervical ganglion of nerves. Dr. Simpson's treatment has been tried, but we believe without any marked success ; and, like some other plans of his, we fear that the remedies may be worse than the disease. As for Dr. Meigs's theoretical suggestions, we fancy they are likely to remain only in the page on which they are transcribed.

We must refer our readers to a very admirable chapter in Dr. Ashwell's work, on the induction of premature labour in pregnancy complicated with organic disease. Dr. Ashwell has the merit of having extended the adoption of this simple plan of treatment to cases in which fibrous tumours imbedded in the uterine walls coexist with pregnancy; and his principal reasons for doing so are, that the danger arises chiefly from the injuries which the growths themselves sustain, which lead on to their inflammation, softening, and suppuration; and that by shortening the term of gestation artificially, these morbid actions are not so likely to supervene.

"To establish its propriety, it is necessary to prove two or three positions, amongst which, I may especially mention the following:

" " st. That when death occurs, after a labour so complicated, the result is only slightly, if at all, referable to the uterus, which rarely sustains any serious mischief; but is mainly produced by inflammation, softening, and unhealthy suppuration in the growth itself; - - these pathological changes leading, in some instances, to rapid sinking; while in others, the powers of the system having been less impaired, death ensues in a few days, from the constitutional collapse, induced by the protraction and difficulty of parturition, and by the contusion and injury done to the tumour and other soft parts. And, 2dly, that premature parturition, artificially induced, rarely occasions constitutional mischief, is easily accomplished, and affords the best, and in many instances, the only chance of a safe result to the mother." (p. 323.)

There are some interesting cases appended to this chapter, which is altogether one of the most instructive and original in the work.

Cancer of the uterus. We shall not follow our authors into their description of this disease; but refer only to their opinions on the important subject of its curability. Dr. Montgomery, it is well known, entertains the belief that cancer in its early stage, when the hard deposit has collected around the glands of the cervix, elevating them into shot-like bodies, may be cured; the principal remedies being rest, abstinence from sexual intercourse, mercury to touch the gums, iodine both internally and externally, and local depletion. On this point Dr. Ashwell concurs generally with him. Dr. Meigs's opinion is thus stated :

"I have certainly met, in the course of thirty years, with several cases of diseased uterus, which I had the greatest reason to suppose cancerous, but which yielded to persevering treatment, and ended in the perfect recovery of health.

"Far be from me the intention to proclaim that 1 have been more fortunate than my brethren, and that I have cured cancer of the womb. My desire is to say, that I was mistaken in my diagnosis, and that I treated a curable and not an incurable malady. I am of the opinion that everybody holds on the subject of this terrible evil, viz. that it is one of the opprobria medicorum, and that it cannot be cured." (p. 281.) 
Dr. Meigs believes that the deposit of the malignant cells is preceded by chronic inflammation and induration. "I cannot bring myself to believe," says he, "that cancer ever commences anywhere as a punctum saliens of disease. There is always an antecedent state of alteration of tissue; a state which lays the foundation for the wild evolution and increase of cells, out of and beyond the control of the accretive and waste laws of the part or organ."

On the employment of iodine in the early stage of cancer, Dr. Ashwell says " that the external application of this drug to the cervix is sufficient to secure its beneficial effects, especially when the friction is persevered in for ten or twelve minutes."

We have ourselves added the italics to this injunction; as we feel bound emphatically to express our dissent from this mode of employing iodine. We should decidedly object to rubbing a stimulating ointment on a part, which, to be cured, requires to be relieved from every source of excitement and congestion; and it appears to us that the act of friction for ten or twelve minutes is sure to excite the aphrodisiac sense, and that, more than probably, it might engender revolting and pernicious habits. It is hardly necessary for us to do more than allude to this ; and it is only the sanction which a name of Dr. Ashwell's respectability gives to the practice, which prevents our using stronger language in discountenancing it. Surely Dr. Ashwell may obtain the full effects of the local application of iodine, by the simple prescription of vaginal suppositories.

On excision of the neck of the uterus. Dr. Ashwell yields apparently a reluctant and rather wavering assent to the occasional use of this expedient in early cancer; " opportunities may occasionally present themselves for its justifiable performance." Dr. Meigs, on the contrary, concurs in the expression of an eminent surgeon of Philadelphia: "If the cervix was cut off, and the woman recovered, it affords the most incontestable proof that the operation was unnecessary." We believe that those who have either witnessed or performed the operation of the entire removal of a carcinomatous cervix uteri, will generally be very indisposed to repeat it; as it is one difficult to accomplish, dangerous from the attendant hemorrhage, and rarely, if ever, successful. On the propriety of attempting to remove the whole womb, it need only be observed, that Velpeau collected twenty-one instances, of which not a single one was permanently cured.

We have so lately commented at length on ulcerations of the os and cervix uteri, in our notice of Mr. Whitehead's book on Abortion, that we have no need to refer to the chapters on this subject by our authors. We merely transcribe, without comment, the following opinions of Dr. Ashwell, in opposition to those of Dr. Bennet:

"In concluding this part of the subject, I may remark that Dr. Bennet regards 'ulceration of the cervix as common with pregnant women.' This, probably, if at all, is only true of the lowest class of females. My own experience is entirely opposed to such an opinion, as well as to another of the same author (pp. 37-8), 'that laceration followed by ulceration, is a frequent occurrence in the first stage of labour,'-a statement entirely at variance with the acknowledged fact, that nature has the power to effect the completion of her own work. Nor can I regard with more favour the conclusion, 'that the state of pregnancy predisposes to inflammation and ulceration of the cervix uteri.' It may be so in prostitutes, and patients suffering from primary and secondary syphilis, in whom pregnancy is happily not a 
common event; and perhaps it may not be regarded as savouring too much of criticism, if I say, that many of the observations of French writers, and even some of our own countrymen who adopt the sentiments of the Parisian school, must be received with great caution in these matters. They are too prone to generalize from the observations they are permitted to make on one section, and that the lowest, in female society. Thus it happens, that the morbid peculiarities of women of abandoned habits, are not infrequently regarded as attaching to the far more numerous class, with whom they have nothing but their sex in common. Hence, also, the too indiscriminate, and often injurious use of the speculum,- the abuse of which has thus far delayed its necessary and justifiable adoption in this country." (p. 433.)

Amongst the affections of the mucous membrane of the cavity of the uterus, Dr. Ashwell includes physometra uteri, a disease which he believes may exist as a primary affection, " the air being secreted by the extensive branches of the uterine vessels ;" although he has never met such a case. Dr. Meigs, who has devoted a letter to the subject, is perfectly incredulous about it; and the first paragraph of the letter is so characteristic of the author's unambiguous way of expressing his opinion, and in our minds so perfectly true, that we cannot avoid copying it :

"Gentlemen,-If you will look into the books, or listen to the relations of your patients, you will, perhaps, be led to believe that the womb is occasionally to be found distended with air, which, after having caused it to expand, until it attains the size of a womb six months gone with child, more or less suddenly escapes; whereupon the signs of the woman's pregnancy disappear, to the great astonishment of the hopeful patient, as was the case in the celebrated instance of Mrs. Commodore Trunnion, of whose baby the author said, 'tenues in auras evadit.' These ventose pregnancies are nonsense, and no thoroughly-bred, and close-thinking physician ought to be for a moment misled by such a story. It is against physiology; it is against pathology; and it flies in the face of common serise, to talk of collections of wind distending a material like the womb, a material which creaks under the edge of the bistoury, and expanding it like a normal ovum, whose gentle slowness of growth is the sole reason for the deployment of the gravid uterus." (p. 288.)

Vesicular moles or hydatids of the uterus. This disease, which is now well known to be a transformation of the tufts of the exochorion, is allowed by Dr. Ashwell to exist in women, quite independent of impregnation or sexual intercourse. Dr. Meigs, on the contrary, affirms, "that he has never heard of hydatids in the virgin, nor does he believe that a virgin could have an hydatid or anything like it developed in her womb." It is perfectly obvious that, if the exochorion be formed upon or from the zona pellucida of the ovum (as Bischoff maintains), as the result of impregnation, it is quite impossible that an hydatid degeneration of it can occur in a virgin. Dr. Ashwell is indeed constrained to have recourse to a membrane, which he calls "opaque, and similar to the decidua, and connected to the uterine vessels," to account for them. We fear that the doctor is hardly aware that he is describing the altered mucous membrane of the uterus; which as a dysmenorrhoal membrane he rather scoffs at, but which he unconsciously admits, and really describes pretty accurately, when he wants it for another purpose. If there be any such bodies as vesicular moles in the virgin, they unquestionably result from the expansion of the decidual glands, broken up into fragments, and expelled with some menstrual blood from the womb. We have ourselves seen such 
masses; but they cannot for one moment be confounded with the dilated exochorion cells, which form the well-known hydatid mole.

In the prognosis of this disease, Dr. Ashwell speaks of the exhausting effects which may result from the attendant hemorrhage; but he does not notice the possibility of death from internal hemorrhage without a trace of bleeding externally. We have ourselves witnessed an instance of the kind, in which a female died with all the signs of internal hemorrhage ; on examination, the uterus, which was supposed to be quite in an early state of pregnancy, reached as high as the umbilicus, and formed a tense hard sac, rather triangular in shape from the stretching out of its cornua; and on opening it, the cavity was quite filled with a mass of hydatiniform exochorion tufts, floating in a large quantity of partly fluid and partly clotted blood, none of which had passed the os uteri internum. The uterus, however, very rarely fails to expel these bodies.

Displacements of the uterus. The displacements of the womb, downward, backwards, and forwards, with its inversion, are severally described by the authors.

Prolapsus uteri. We shall only refer to this displacement, which is fully treated of by Dr. Ashwell, and very graphically described by Dr. Meigs, to allude to the employment of the pessary, which is advocated with positive enthusiasm by Dr. Meigs, and with a very strong predilection for it by Dr. Ashwell. The latter author says that he has used it for the last twenty-six years, and that "he has met with very few instances out of a great number where it could not be introduced, and none where its employment occasioned permanent mischief." Dr. Meigs's allusion to it in the following paragraph, is to the purpose, and is so characteristic of his style that we are induced to transcribe it :

"As to my own preferences in the matter of pessaries, I have only to say, that whatever may keep the womb at its due height without irritating it, or incommoding the other organs and textures implicated in the descent, is a good pessary; but I deem the globe the most perfect and most suitable for the ordinary simple cases. An instrument of two inches, pressed upwards to the uterine extremity of the vagina, lifts the womb high enough ; higher, in fact, than the position occupied by it in eight out of ten of those women who have had a child, and that is high enough. It is kept up by the double and consentaneous actions of the sphincter vaginæ muscle, and the levator ani. It has no angles, no sides; it cannot be displaced, save by being ejected. Its pressure is uniform over its whole superficies, save where its lower segment looks down the tube of the vagina. This cannot be so truly said of any other instrument, if we except, perhaps, the gum-elastic bottle, used and recommended by Dr. Hervez de Chegoin, in his paper in the 'Mém. de l'Acad. Roy. de Méd.' The globe has a perfect polish, and an unoxydizable surface. It may be worn a year or more without displacement, if required; and it has no aperture to admit of the collection of putrescible materials within it. It does not prevent the escape of the mucus of the uterus and vagina, nor of the menstrua; and, in short, is the least uncomfortable and most perfect of instruments. It is a true suspensory, as needful for the descended womb as the suspensor scroti for a hernia humoralis or orchitis. It is as neat and perfect in its kind, as Petit's tourniquet in its kind, and as indispensable for the cure, Dr. Physick used to tell us in his Lectures, that a man might as well attempt to improve the Bible as Petit's tourniquet, and I, after him, repeat, that you might as well attempt. to improve Petit's tourniquet, as Physick's globe pessary. But you know I always have warned you to be Nullius addictus in verba jurare magistri, and so I say, judge of it for yourselves." (pp. 172-3.) 
We must say, that personally we do not sympathise with the authors in their excessive admiration for pessaries ; and we are not a little surprised to hear that Dr. Ashwell has never known them do permanent harm. We are quite sure that neither of them has given weight enough to the fact, that partial descent of the womb is a very common result of congestion and hypertrophy of its body and neck; and that a weakened vagina is by no means so exclusive and proximate a cause of it, as Dr. Meigs professes to believe. We have seen pessaries of box-wood and caoutchouc of various shapes, used very extensively; and they have left on our minds the impression that they are clumsy, filthy, and odious contrivances, exciting great irritation in the cervix uteri and vagina, followed frequently by fetid discharges. They are often very difficult satisfactorily to adjust, and sometimes more difficult to remove; and we quite concur with the late Dr. Hamilton in believing that they rarely produce, even at the best, more than a palliative effect. And in proportion as in practice we have discarded the use of vaginal pessaries, so have we adopted a form of external support, in the shape of a more or less deep perineal pad, fixed to the edge of an elastic abdominal belt. That this is not equal to keeping up an uterus which has long been procident, or which, from the excessive relaxation of the sexual passages, becomes so on the slightest exertion, we freely admit. In such cases, a well-made stem-pessary, such as that described by Dr. Meigs as Blundell's pessary (vide p. 181), will be found necessary. But our observation applies to the general run of cases of prolapsed uterus ; and we confidently affirm, in spite of Dr. Meigs's pedantic theorizing, ${ }^{*}$ that very great relief, and, with the concurrent aid of astringent applications, frequent permanent cures, are effected by them.

It is certainly very perplexing to notice the diversity of opinion which two practical physicians, like the authors, entertain occasionally on the use of the same thing; and in illustrating this, we would refer to their views on the sponge pessary. Dr. Ashwell says of it: "I know only of one case in which it should be used,- - where there is excessive irritation and tenderness of the vagina ;" while Dr. Meigs " protests against the sponge -most particularly against the sponge." - "I protest," says he, "against its employment as a pessary, not only on account of its irremissible fault of uncleanness, but for the additional objection of its irritating nature ; the points of the sponge could not fail to vex and fret the mucous tissue of the vagina." We must say that we have always found it a most irritating substance; and we should, from our own experience, make a point of excluding it in the cases which Dr. Ashwell has selected for its employment.

Dr. Meigs assures us, from repeated observations, that the globe pessary does not prove a bar either to conception or gestation.

Retroversion of the uterus. This accident, as an accompaniment of pregnancy, has been known and described by authors since Gregoire and Dr. William Hunter first revealed its nature; and there is nothing in the opinions of the authors concerning this particular complication, which requires notice. Retroversion of the unimpregnated uterus is a subject of some interest at the present time, on account of the attention which, by several writers, has lately been directed to it. And here again we have a discrepancy of opinion, not only between the two authors, but we may

* Vide Observations against the Use of Utero-Abdominal Supporters, p. 188. 
also say, amongst obstetricians generally. Dr. Meigs speaks of it as one of the "most common of the deviations of the womb met with in practice;" while Dr. Ashwell censures M. Lisfranc as a man indulging largely in the marvellous, when he endeavours to show that it is a frequent displacement of the unimpregnated uterus, resulting from hypertrophy of the posterior wall. Dr. Ashwell speaks of it "as almost entirely a disease of early pregnancy ;" and yet, with an inconsistency which, after his lavish censure of M. Lisfranc, is very apparent, he says :

"I believe partial retroversion of the unimpregnated womb to be a more frequent occurrence than is generally supposed; its most common cause being hypertrophy, or more serious disease, of the posterior wall of the organ. If in such cases, where there is difficulty of micturition and defecation, a careful examination were made, the uterus would, I am persuaded, be often found more or less retroverted." (p. 626.)

We can quite understand Dr. Ashwell's habitual caution in receiving statements from M. Lisfranc, after the story which is abroad of his cases of excision of the cervix uteri ; but we suspect that in his estimate of the frequency of retroversion, this very intelligent surgeon has not gone beyond the truth; and that our present knowledge on this subject will be found to corroborate it. For our own parts, we perfectly concur with M. Lisfranc in thinking that it is so frequent a displacement, that a man who has had large public opportunities of examining uterine disease, may in a few years number up, without the least exaggeration, his hundreds of cases. And we further concur with him, that it is a large bulky womb, with the back wall particularly prominent, which is the most common cause of this deviation. It is certainly important to remember, especially when considering the numerical frequency of the complaint, that retroversion, like the descent downwards, has different degrees ; and we particularly insist on this, as Dr. Meigs has supplied a drawing in which an unimpregnated uterus is completely retroverted, its fundus being quite low down in the recto-vaginal pouch, and the cervix looking directly upwards in the axis of the brim of the pelvis. If. Dr. Meigs means this to be the practical representation of an unimpregnated womb in the ordinary extent of retroversion, we do not hesitate to say, that it is the rarest of uterine deviations. Retroversion is only a common complaint, when it occurs in a far less marked degree; the fundus falling backward towards the upper part of the sacrum, and giving the womb as much an inclination backwards, as in its normal position it would be directed forwards. This observation, we know from our own experience, applies also to a womb retroverted in early pregnancy; and the cases related by Dr. Ashwell, which required the introduction of the entire hand into the rectum, to get bearing enough on the bulging fundus uteri to raise and restore it, are extreme cases but rarely encountered; whereas a less decided retroversion is more frequently met with amongst cases of abortion (to which it is related as a cause) in ordinary practice.

Dr. Meigs labours to prove that the cause of retroversion, "the maladive or pathological fault, is in the round ligaments; and not in the womb itself." He does not appear to have noticed the overweighing of the womb, which, from affecting the posterior wall principally, inclines the whole organ backwards, and, as a secondary effect, puts the round ligaments on the stretch. His treatment of a case is to raise the womb by 
a vaginal pessary so as to relax the round ligaments, which will then contract to their proper length and recover their tone. We, however, who accord with those who regard the womb as primarily in fault, (with but few exceptions), consider that the first indication to be fulfilled is to reduce its size, and then to restore tone to the weakened structure.

The opposite displacement, viz. anteversion, is regarded by both the authors as a very uncommon disorder. Dr. Meigs has only seen three or four cases of it in the course of his professional life. Dr. Ashwell relates a case furnished to him by Dr. Walshe, in which the uterus was found after death to be "flexed on itself, at an obtuse angle, at the union of its body and neck, in such a manner that the fundus concealed by the bladder was inclined forwards and downwards, while the neck was inclined backwards to the sacrum, the posterior surface of the body being antero-superior." "The body of the organ, as well as the neck, is hypertrophous; their substance is of a grayish hue, and hardened, firm, and resisting throughout, except at the union of those parts, where there is a band of the organ flattened from before to behind, extremely soft, flabby, and yielding, and corresponding exactly to the angle of flexion."*

This interesting case, which was reported by Dr. Walshe from a patient of M. Louis, of Paris, is one in which the flexion of the womb forwards is more decided even than its anteversion.

Flexions of the uterus. - The two displacements of the uterus which we have just noticed, are connected with those bends of this organ which have respectively been called Anteflexion and Retroflexion, and which have been reported by some recent writers to be of very frequent occurrence. We looked with some interest at the opinions and experience of the authors on this subject; and it is satisfactory to find that there is a general accordance in their views. They both regard these flexions as part only, and an unimportant part too, of some displacement of the uterus, whether prolapsus or retroversion; although Dr. Meigs does not deny but that a congenital cause, such as an unequal development of the cervix, "causing the anterior or posterior half to be too short," may occasion them. "Retroflexion," says Dr. Ashwell, "may be regarded as partial retroversion, the same in every respect save one-the alteration of position of the cervix, which, from preserving its proper locality, renders curvature or flexion of the body necessary to the production of the displacement."

"Many women," says Dr. Meigs, "have the cervix uteri very slender; some of them are not bigger than the little finger. Now if a woman with such a slender, flexible, and weak cervix, should have some degree of descent of the womb, it might happen that the long slender cervix, being driven, or having descended far enough to rest, upon the posterior part of the pelvis, or even on the posterior wall of the vagina, should bend with its own weight and any superadded weight, so that the cervix would cease to be straight, but become curved or angular; and after being kept so for many months, would acquire such a form, as its permanent character." (p. 224.)

Our own observation as to these flexions is quite in keeping with the above-quoted opinions ; and excepting only those curious cases in which the womb is congenitally twisted, or in which, as in Dr. Walshe's case, there has been a loose, thin, flaccid texture at the junction of the body and neck of the uterus, allowing the former to fall or be drawn 
forward, we think the flexion might well be merged in the graver displacement in which it partakes. like Dr. Meigs, we "do not know that these curved or angular conditions of the cervix are, in themselves considered, to be esteemed as matters of any great consequence ;" and we are well persuaded that those who have written in the periodical journals so much about retroflexion, might just as well have avoided complicating the real disease, viz. retroversion of the body of the uterus, by the introduction of the title. It may no doubt be very entertaining, and wear a specious appearance of originality, to elicit new diseases from those which are clear and well understood : and we quite accord with Dr. Ashwell, in believing that " many cases of slight and unimportant uterine displacements have been most erroneously set down as examples of the anterior and posterior flexion."

The diagnosis and management of these versions and flexions of the unimpregnated uterus, have been said by Dr. Simpson, and several other obstetric practitioners, to be greatly facilitated by the use of the uterine sound, and by a particular contrivance invented by Dr. Simpson for maintaining the uterus in situ. The lavish encomiums which have been expended on the former instrument by these gentlemen, have, we believe, caused it to be pretty extensively employed in diagnosing these affections. By introducing the sound into the cavity of the uterus, the direction of the body of the organ is indicated; and when once within the womb, it can be made to restore the displaced organ, or to carry it from side to side, or to remake the displacement at will. All this may be done, it is confidently said, without any evil resulting from it. We have already said something on this subject, in our allusion to the sound as a means of diagnosis in uterine disease; but we cannot help expressing our cordial concurrence with Dr. Ashwell, when, in reference to its application to these particular complaints, he says-

"I cannot avoid thinking, that this uterine sound not only detects, but makes many of these supposed displacements. All practical men know that the uterus varies naturally in its position, in its degrees of mobility and immobility, and in the influence exerted upon it, as to position, by a loaded or empty rectum or bladder; and it must be kept in view, that the curve of this steel bougie may not be the curve of the uterus; and if, therefore, it is to be introduced at all (and I wish it were far less frequently so), the normal position of the organ, thus spiked, must be made to follow the curve of the iron instrument, entering, and thus necessarily intruding upon, its cavity." (p. 635.)

And then, again, we find Dr. Meigs agreeing with Dr. Ashwell in deprecating the employment of such an uterine supporter as Dr. Simpson's; the peculiarity of which, as our readers are doubtless aware, consists in an ivory stem, which is passed into the uterine cavity, and is made, by its union with the other parts of the instrument, to be the immediate support to the replaced womb. " Some have proposed," says Dr. Meigs, "to pass up a small spatula of wood (it might be well denominated a peg) into the canal of the cervix, to straighten the bent cervix, by leaving it in situ. Such a process of spitting or skewering the womb appears to me ridiculous."

Will the womb bear this foreign body? Does it not occasion irritation, discharges, direct or contiguous inflammation?-are questions which at once suggest themselves to every practical inquirer; and an anxious desire to hear the worst as well as the best of it, must be present in the minds of all who are disposed to try it. Dr. Simpson's report of it is unexcep- 
tionably good. A gentleman quoted by Dr. Ashwell-Dr. Hensley, has known peritonitis induced through the patient not having been kept quiet in bed for some days after its introduction; while Dr. Ashwell's own experience of it in two cases, is thus described:

"Two cases have recently come under my care, and I have heard of more, where the results arising from the use of this intrument have been very serious. Looking at it pathologically, I can scarcely imagine anything better devised for inducing disease. According to this practice, a piece of ivory, two inches and a half long, is to be introduced into the uterine cavity, and its bearing must of necessity be on some part of the lining membrane, a surface ill adapted to support the pressure for two or three months together of such an instrument. The consequences may be supposed. One of the patients from whom, after a long and distressing journey, I removed this ivory one-pronged fork, told me she had never been free from pain since its introduction. In addition, it had produced frequent and intense sexual excitement, preventing sleep for many nights together, and had kept up constant leucorrhœal discharge. In the other example, during the two months this instrument had been worn, the sufferer, in addition to the previous complaints, had never been free from sanguineous discharge, lumbar pain, and frequent desire to micturate. In both, the speculum showed that abrasion of the os and the ostium vaginæ had resulted from the use of the so-called uterine supporter." (p. 636.)

Our own experience of the practical value of this supporter is certainly unfavorable. It is sometimes difficult to adjust; and the irritation which it sets up we have found to be great and formidable. There is much difference, no doubt, in its effect on the uterus of different women, some tolerating it comparatively well; but at the best, we confidently believe, there is much risk run of immediate or remote disorder. Within three weeks of the time we are writing, we saw a young unmarried female, whose previous history and disease we were already acquainted with, and who some months before had had the ivory stem passed into the uterus by one of that docile flock of London obstetricians who bow their ear obsequiously to the tinkling of their northern leader's bell, and who applied to us in consequence of a return of the same symptoms as those from which she had previously suffered. The uterus was slightly prolapsed and partially retroverted; but, as usual in such cases, it was easily restored by pressing the bulging back wall upwards and forwards. For this the sound had been passed, and then the ivory-stemmed supporter. The immediate effect of the latter was to bring on severe pain and fever, which was followed by cellular inflammation and suppuration, the large cicatrix of the opened abscess being visible on the abdominal integument on the left side. When she recovered from this, however, the stem was again passed, and at last she was able to bear it; and the restoration of the womb gave her relief from her lumbar and bearing-down pains. She wore this stem for several weeks, and when it was removed she was thought to be cured. The symptoms, however, soon again returned, and were both aggravated, and in a great measure reproduced, by the habit of masturbation, which she had acquired since the application of this support. The os uteri of this young woman is open enough to admit the tip of the index-finger.

There is an asperity, and a tone of ironical contempt, about Dr. Ashwell's remarks on both the uterine sound and this supporter, which can hardly be justified, excepting on the supposition that he believes folly is mixed up with their invention, and rashness with their use. If not, they deserve 
a more respectful notice, and a more deliberate trial. We feel, too, that these incidental remarks suggest a question which his own sagacity has partially brought to bear to the present complaints, and which may well be made to include other methods of treatment in other diseases of the womb. Is there anything so serious in a retroversion or retroflexion of the unimpregnated uterus, as to justify a practitioner in running the risk of exciting peritonitis, cellular inflammation, and abscess, -injuring the structure of the womb,-and enkindling desires, or implanting habits, which destroy purity of feeling and physical health? Our own conviction is, that this supporter will be found to create more disease than it cures; and with the belief that the uterine sound will be extensively because easily employed, the same observation will, we think, be verified in it. The habitual use of powerful escharotics in simple inflammatory hypertrophy of the cervix, cicatrizing and contracting the os and cervix, and frequently too the vagina, and risking deeper-seated pelvic inflammation, is open to a similar inquiry, and the same animadversion. May we not add, too, the practice, now becoming common, of opening and dilating the os uteri, whenever it happens to feel rather small, and smearing the uterine cavity with the solid nitrate of silver, to excite the menstrual flux; and, at last, to cover the spirit of obstetric adventure with the mantle of Quixotism, the announcement that the Fallopian tubes are to be explored to remove sterility? Surely, in all these, there is much that is visionary; more that is meddling; and in all there is the tendency to create more disease than they cure. It appears to us that much of what has been proposed and adopted in the treatment of uterine disease within the last six years, has been empirical, and rashly experimental; and that the evil results have been in a great measure concealed. Those who see the greatest diversity of practice,-men like Dr. Locock, Dr. Ferguson, Dr. Lee, \&c.,-ought, as a debt which they owe to their profession, to speak out upon these matters; and we do not think we anticipate too much in believing, that they would condemn much that we have ourselves censured.

Diseases of the ovaries. Acute and chronic ovaritis, dropsy of the ovary, atrophy of the ovary, fibrous tumours and malignant diseases of the ovary, are described by Dr. Ashwell; and a letter on these diseases is supplied by Dr. Meigs.

Dropsy of the ovary. So much interest has of late been excited about this disease, from a revival of the formidable operation for the extirpation of the cyst, that we are glad to have the opinions of two practical physicians like the authors on the subject. They both, however, agree in condemning it. Dr. Meigs acknowledges the fact, which Mr. Lee's tables seem to prove statistically, that tapping the cyst is far more frequently followed by death, either within a few hours or a few weeks, than might have been anticipated; and that therefore, although simple enough in one sense as an operation, it is dangerous in its issue. Dr. Meigs states his own impression to be, that of first tappings, of which he has been witness, nearly one half have been speedily followed by the death of the patient from peritonitis, developed soon after the performance of it; and all, with one exception, soon ended fatally. To this we may also add another fact elicited from Mr. Lee's tables; viz., that there are on the average three recoveries to one death after ovariotomy; and that the results of other capital operations in surgery yield a greater mortality. Dr. Meigs, how- 
ever, thus summarily states his objection to it, and disposes of the argument derived from its comparison with other operations :

"I am opposed to the operation for extirpating the diseased ovary. The ground of my objection lies in that principle of surgery, which I hold to be one of its highest principles, that there can be no duress, no binding obligation to perform it, and that the surgeon's conscience may be at peace, though he refuse and the patient perish by the progress of the malady.

"I have read with great pleasure Professor Simpson's remarks in favour of ovariotomy, in a pamphlet, for a copy of which I am indebted to his politeness. I admit the considerable force of many of the arguments arrayed in Professor Simpson's publication, but I see no parity in the duress of the surgeon who decides upon an ovariotomy, and that of the surgeon who performs the operation for the stone, or an amputation, or the ligature of an important vessel, \&c., which he dare not refuse to perform, whereas no such urgent, imminent, and definite necessity can ever be supposed of an ovariotomy operation.

"In the one case, the surgeon resorts to the operation under compulsion of an imminent and pressing danger; in the other, he takes his instrument in his hand under the dictation of a cold calculation of chances as to a human life. Now who, I pray, has the right to calculate as to the value of a man's life, even for an hour, much more for a week, a month, a year, or a quarter of a century !

"I am opposed then to the operation of ovariotomy, and I am opposed to it on the grounds of objection I consider valid against all surgery that is not unavoidable.

"The ovariotomy operations are not unavoidable, since no one, I presume, would think of operating for a patient not remote from dissolution, and since, in any case not menacing a speedy termination of the life of the patient, the unavoidable necessity has not arrived." (pp. 313-14.)

Dr. Ashwell is also very strongly opposed to it, and considers that it will ever remain an eminently uncertain operation. Still he appears to admit that it may sometimes be justifiable, and he limits it in the following manner :

"If the operation is to come into established practice, of which I have the strongest doubt, it must be confined to examples of the malady where tapping has already been so often performed as to preclude, from the experience of similar cases, any idea that it can ever be dispensed with; and where, we are confident, that great suffering must lead to early death. Perhaps this may be regarded as too limited a view of the value of extirpation; but it is, I think, the correct one. In such cases, if the diagnosis excludes the belief that there are serious adhesions, or malignant and solid growths complicating the tumour, and if the patient strongly desires it, the operation is defensible." (p. 663.)

We cannot say that the principle on which Dr. Meigs acts, in altogether excluding ovariotomy, is valid or decisive. It has, perhaps, a limited application to early cases, when the size of the tumour has not interfered mechanically with important organs; and any such operation exposes the subject prematurely to the serious risks attending it. But when the disease has been of long standing, and is steadily increasing, and our ordinary resources have proved but feeble palliatives, - when its bulk has begun to encroach on the thorax, and the swelling of the abdomen is the source of incessant distress, and is a hinderance to the performance of the duties and enjoyments of life, - when we feel the practical assurance that its further increase must destroy life, and the patient (being apprized of the risks) is anxious, often urgent for the aid, and there is no forbidding complication, either in the probable attachments of the tumour, or malignant disease elsewhere, or other organic disease,- - surely, under such circumstances, it 
becomes an unavoidable operation, the conscience of the surgeon making it so ; unless, indeed, this has been rendered insensible to the stigma of remissness and neglect, by the extreme adoption of an arbitrary principle. There is a reasonable expectation that a patient so circumstanced may not only be released from distressing suffering, but may literally be restored to perfect health. The statistics even give three recoveries to one death. And even allowing that this result is too favorable, it is impossible to have witnessed the issue of favorable cases, as we have had the good fortune to do, and not to feel that surgery has had a perfect triumph, in rescuing the subjects of this disease from impending death, and from sufferings and social privations almost worse than death.

It is beyond our purpose to enter in detail upon the diagnosis of the disease itself, and of adhesions around the tumour, as bearing on the feasibility of the operation ; but we feel well persuaded that the eager attention which has been paid to these points will greatly diminish the uncertainty concerning them. The careful preparation of the patient for the operation, and the attendant circumstances of temperature, \&c., during it, all contribute greatly to its success ; and it is not, we think, anticipating too much to believe, that a watchful experience will render abdominal surgery generally far less formidable, and far more successful, than it has hitherto been.

There is a brief account of the diseases of the external organs, by the authors; and a short notice, in Dr. Ashwell's work, of a painful affection of the urethra, which he has termed chronic urethritis. We know something of the class of cases to which he refers, and fully concur with him as to their intractability, and the extreme distress which they occasion. Whether they are truly the result of chronic urethritis, may perhaps be questioned. We have examined the urethra with a speculum, in such cases, without detecting any appearance which could be denominated chronic inflammation; there is no purulent discharge; and local applications have been of little avail. We quite accord with Dr. Ashwell in the expediency of a mild mercurial course in this affection. We have known the disorder, in one instance, disappear suddenly, after having. resisted a variety of treatment for a length of time, apparently from the action of a strong purgative. Dr. Meigs's letters on the diseases of the external organs, contain many interesting and rare cases, and many instructive observations.

In bringing our notice of these works to a close, we have only to remark, that our criticism of them has been in a measure proportioned to the high reputation of their authors. We think that Dr. Ashwell's work will bear a careful revision, and some condensation. The cases require to be much curtailed, and their points brought out by the author himself. It would be all the better if some of them were incorporated with the text, in connexion with the subject which they immediately illustrate, and many may be sacrificed altogether. There is a good deal of the haste of a busy man in the work; and here and there an observation occurs in one part, which is out of keeping with another subsequently made. The author, too, is sometimes circumlocutory, almost obscure, in the statement of his opinions; and, when this is the case, it requires the reader to reperuse the paragraph again and again, before it is quite intelligible. If the author, in the next edition, would bestow some time in revising the present volume, we are 
quite sure that he would soon weed it of these faults. We take our leave of Dr. Meigs, with a high opinion of his talents and originality; and we trust that, if we meet him again as an author, we shall have to notice his solid, substantial merits only, without such an obtruding, meretricious dress, as he has chosen in his present Letters.

\section{ART. IX.}

On Femoral Rupture; its Anatomy, Pathology, and Surgery. With a new Mode of Operating, applicable to Cases of Strangulated Hernia generally. With Plates. By JoHN GAY, F.R.c.s., Surgeon to the Royal Free Hospital, \&c.-London, 1848. 4to, pp. 97.

WE opened this new quarto with considerable interest, being curious to know what could possibly be written new, on a subject so exhausted as that of hernia - a subject so fully considered in all our surgical dictionaries, manuals, and lectures, and in the numerous special works which its importance has called forth. The interest was not diminished by the statement in the preface, that the author proposed to describe the anatomy of the parts concerned in femoral rupture, both before and after its formation, "from their careful examination in above seventy subjects ;" and that from this study of anatomical relations he had been led "to relieve the mass when strangulated by a new method of operating, which I believe to be more simple and safer than any which has hitherto been practised or described;" the principle of which is capable of being applied to the relief, not only of femoral, but to all outward forms of strangulated hernia. This appeared very promising; but on carefully going over the first half of the book, we found to our great disappointment, that the dissection of the seventy subjects had led to nothing more than such a description of the surgical anatomy of hernia, as any student might compile from the works of Cooper, Lawrence, and Hey, with an occasional reference to Cloquet; the descriptions not being remarkable for clearness, nor the language for correctness-although both are free from any errors sufficiently glaring to call for especial notice. Even where one would expect the seventy dissections to necessarily yield some results, as in the account of the normal and abnormal courses and branches of the obturator and epigastric arteries, and of the different measurements from the centre of the sac to the orifice, the rings, arteries, and other neighbouring parts, Mr. Gay says not one word from personal observation. His measurements are all taken from Sir Astley Cooper, and his anatomy of the arteries from Quain, Harrison, and Burns : but we learn nothing of the statistics of the seventy cases here, or indeed anywhere but in the preface. We are not told where the seventy subjects were so opportunely obtained, where the dissections were made, where the dissected parts are preserved, nor, as we said before, can we find a single result of original research in the whole of the anatomical portion of the book.

A few observations follow, equally destitute of novelty, upon strangulation of hernia, and on the ordinary and unusual seats of stricture; and then we come to the surgical part of the work, which is commenced, singularly enough, by a short account of some of the different measures proposed for the radical cure of hernia. This section is concluded by the following extraordinary sentence. 